Ergastoplasma-Reticulums und besitzt optisch leere Zisternen. Die Mitochondrien weisen nur geringe Schwellung auf. Der Quarz liegt auch hier frei im Cytoplasma. In Abb. 6 ist eine freiliegende Zelle mit Quarzeinlagerungen wiedergegeben; die Kristalle besitzen muscheligen Bruch und waren in dieser Art seltener als die länglichen Splitter anzutreffen. Die Mitochondrien sind nicht geschwollen, woraus geschlossen werden könnte, daß möglicherweise der hier gespeicherte Quarz längere Zeit benötigt hätte, um in der Zelle cytotoxische Wirkungen zu entfalten.

Wir schulden Dank Herrn Dr. H.-F. Esser für die Hilfe beim Tierexperiment, Herrn Dr. I. Langhoff für die Oberflächenbestimmung und den Herren Dipl.-Min. S. Pollmann und Dr. R.-W. Schliephake für röntgenographische Quarzbestimmungen. Durchgeführt mit einer finanziellen Beihilfe der $\mathrm{H}$ ohen Behörde der Europäischen Gemeinschaft $\mathrm{für}$ Kohle und Stahl.

\title{
Chinone als aktives Prinzip der Abwehrstoffe von Diplopoden
}

\author{
Von H. SchildkNecht und K. H. WeIs \\ Aus dem Institut für Organische Chemie der Universität Erlangen \\ (Z. Naturforschg. 16 b, 810-816 [1961]; eingegangen am 12. August 1961)
}

\begin{abstract}
Als Inhaltsstoffe der Abwehrblasen von Cylindroiulus teutonicus Pocock, Brachyiulus unilineatus Koch und von noch nicht genau bestimmten Diplopodenarten aus Belgisch-Kongo, Neu-Guinea und Madagaskar konnte uv-absorptionsspektroskopisch bzw. durch adsorptions- und papierchromatographische Untersuchungen der 2.4-Dinitrophenylhydrazin-Fällung Toluchinon und 2-Methyl-3-methoxy$p$-benzochinon identifiziert werden. Die je Individuum in den Blasen gespeicherte Chinonmenge beträgt bei den einheimischen Arten ca. 1,2 mg und bei den fremdländischen ca. 200 bis 350 Milligramm.
\end{abstract}

Das Vorkommen von Abwehrstoffen bei Diplopoden war schon zu Beginn des vorigen Jahrhunderts bekannt. 1878 beschreibt Voges ${ }^{1}$ Lage und Bau des chemischen Wehrapparates am Beispiel des Spirobolus cupulifer V. Es befinden sich an beiden Körperseiten in ununterbrochener Folge d.h. in jedem Ring die Foramina repugnatoria (F), Mündungen retortenartig gestalteter Beutel (B), die den Wehrsaft enthalten (s. Abb. 1).

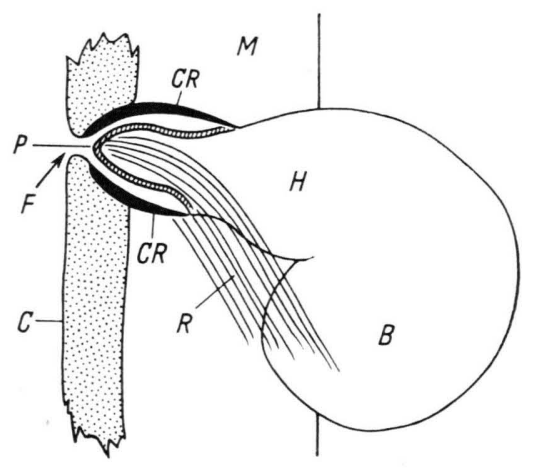

Abb. 1. Die Vorratsblase für den Wehrsaft eines Diplopoden. $\mathrm{B}=$ Blase; $\mathrm{H}=$ Blasenhals; $\mathrm{M}=$ Körpermuskulatur; $\mathrm{CR}=$ Chitinrinne; $\mathrm{R}=$ Rückziehmuskulatur; $\mathrm{P}=$ Pfropfen $; \mathrm{F}=$ Foramen repugnatorium (Austrittsöffnung); $\mathrm{C}=$ Chitinpanzer.
Der Hals (H) der Blase (B) durchstößt eine Schicht der Körpermuskulatur (M), im Bild gesehen von vorne nach hinten, und gelangt so an die innere Oberfläche des Integuments, woselbst er in einen kleinen Porus der Körperdecke überführt.

Die eine seitliche Hälfte des Halses besteht aus einer Chitinrinne (CR), welche an ihrer Scheitelfläche zu einem Pfropfen (P) ausgebildet ist, welcher den Porus verschließt. Ein Muskel, der die Hautmuskellagen durchdringt, inseriert am rinnenförmig ausgehöhlten Endstück des Drüsensackes. Durch seine Kontraktion wird das Chitinstück zurückgezogen und damit auch der Pfropfen, so daß der Porus geöffnet wird. Das Sekret wird durch Kontraktion der die Drüsensäcke umgebenden Hautmuskelschicht herausgepreßt.

Es fehlte nicht an Versuchen, die chemische Natur derselben zu erfassen. Auf Grund eines chlor- oder jodähnlichen Geruchs glaubte man an das Vorliegen von freiem Halogen in den untersuchten Sekreten einiger Tausendfüßlerarten. Holl ${ }^{2}$ versetzte im Jahre 1826 das Sekret von Julus foetidissimus mit

\footnotetext{
1 E. Voges, Beiträge zur Kenntnis der Juliden (1878), s. a. l. c. ${ }^{3}$.

${ }^{2}$ Fr. Holl, Neues Journal der Pharmazie (1826), s. a. l. c. ${ }^{3}$.
} 
einer Aufschlämmung von Stärkemehl und beobachtete eine Violettfärbung, die er beweisend für das Vorliegen von „Joden“ hielt. Auch VaubeL ${ }^{3}$ ist noch 1901 davon überzeugt, Jod vor sich zu haben, obwohl er bei den von ihm untersuchten deutschen Juliden solches in freier Form nicht nachweisen konnte.

Erstmals erkannten BÉHAL und PhISALIx ${ }^{4}$ Chinon als den wirksamen Stoff im Sekret eines Diplopoden. Sie untersuchten den Schnurfüßler Julus terrestris (Schizophyllum mediterraneum), bei dem sie mittels einer Farbreaktion nach LiebermanN ${ }^{5} p$-Benzochinon-1.4 fanden. Seit dieser grundlegenden Entdeckung vergingen 57 Jahre bis BARBIER und LEDE${ }_{\mathrm{RER}}{ }^{6}$ bei Spirostreptus castaneum A t t e m s $p$-Benzochinon-1.4 und bei Spirostreptus virgator Silv. 2-Methyl-p-benzochinon-1.4 neben weiteren unbekannten Chinonen fanden. 2-Methyl- $p$-benzochinon1.4 entdeckte dieser Arbeitskreis auch bei Pachybolus laminatus. Trave und Mitarbb. ${ }^{7}$ fanden 1959 bei Archiulus (Schizophyllum) sabulosus L neben 2-Methyl-p-benzochinon-1.4 schließlich ein bis dahin bei Arthropoden noch nicht bekannt gewordenes Chinon, das 2-Methyl-3-Methoxy-p-benzochinon-1.4. Hierzu gewannen sie das Gift von 1250 Tieren, eine Menge von etwa $1 \mathrm{Gramm}$, setzten mit Dimethylbutadien um und führten die erhaltenen Produkte der Dienkondensation in mehrstufiger Reaktion in die entsprechenden Naphthochinone über, welche sie durch fraktionierte Sublimation voneinander trennten.

Die Verschiedenartigkeit der bei den einzelnen untersuchten Arten auftretenden Sekretbestandteile ließ es wünschenswert erscheinen, mit Hilfe der beschriebenen Mikromethoden ${ }^{8}$ die Chinone einiger Diplopoden verschiedenster Gattungen und Herkunft zu untersuchen, um so einen Überblick über die Abwehrstoffe dieser Tierklasse zu erhalten.

\section{Material und Methode}

$$
\text { 1. Die Studienobjekte }
$$

Die Wahl der Studienobjekte erfolgte lediglich nach einer möglichst weiten Verbreitung der Herkunftsländer. Im übrigen war sie durch leihweise Überlassung der Tiere durch zoologische Institute bedingt. Die ein-

3 H. G. Brons, Klassen und Ordnungen des Tierreiches, V, 2/II, Leipzig - Akademische Verlagsgesellschaft 1928.

4 A. Béhal u. M. Phisalix, C. R. 131, (Nr. 24), 1004 [1900].

5 C. Liebermann, Ber. dtsch. chem. Ges. 10, 1614 [1877].

6 M. Barbier u. E. Lederer, Biochimica 22, 236 [1957]. heimischen Arten wurden auf eigenen Exkursionen beschafft.

Die Haltung erfolgte im Terrarium auf einer Schicht Ackererde, die mit Moosstücken belegt war. Zur Fütterung dienten auf Vorschlag von Herrn Prof. Stammer und Herrn Dr. Osche Apfelstücke und faulende Bananen. Für gute Befeuchtung wurde Sorge getragen.

Im einzelnen haben wir untersucht:

2 einheimische Juliden

Cylindroiulus teutonicus $\mathrm{P}$ o c o c k und

Brachyiulus unilineatus $\mathrm{K}$ o c h

1 Vertreter der Spiroboloideae

Unbekannte Art der Gattung Rhinocricus aus Peru: hellbraun- mit orangeroten Rändern der Segmente; etwa $10 \mathrm{~cm}$ lang (s. Abb. 2).

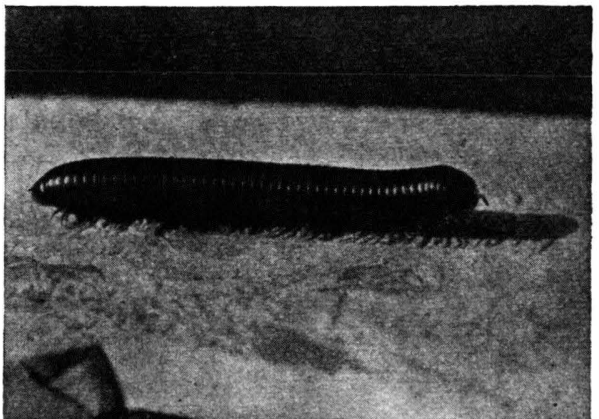

Abb. 2. Der peruanische Rhinocricus, etwa $10 \mathrm{~cm}$ lang.

3 Vertreter der Spirostreptoideae

Unbekannte Art aus Madagaskar : gleichförmig dunkelbraun; etwa $14 \mathrm{~cm}$ lang; unbekannte Art aus Neu-Guinea: gleichförmig grünschwarz; etwa $16 \mathrm{~cm}$ lang (s. Abb. 3); unbekannte Art aus Belgisch Kongo: braun, mit dunklen Rändern der Segmente; etwa $14 \mathrm{~cm}$ lang.

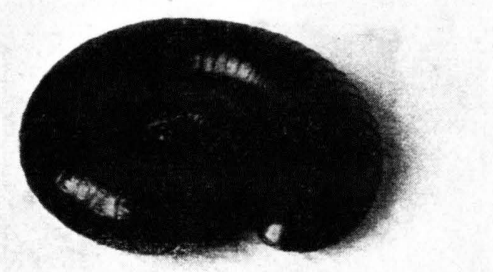

Abb. 3. Spirostreptus-Art aus Neu-Guinea in Schreckstellung.

2. Gewinnung der Sekrete und Analyse Die kleinen einheimischen Arten wurden auf bekannte Weise ${ }^{9}$ abgesaugt, wobei sie u. a. ihr Sekret

7 R. Trave, L. Garanti u. M. Pavan, Chimica Industria 41, 19 [1959].

8 H. SchildKnecht, XI. Internationaler Kongreß für Entomologie, Wien 1960, Verhandlungen Bd. III, 269.

9 H. Schildknecht, Angew. Chem. 71, 524 [1959]. 
beim Betupfen der Körpersegmente mit einer stumpfen Nadel abgaben. Als Vorlage dienten salzsaure 2.4Dinitrophenylhydrazin-Lösung (2.4-DNP), Alkohol oder Äther, je nach Methode der Analyse. Die großen überseeischen Arten wurden am bequemsten in ein Reagenzglas passender Weite eingeschoben und mit einigen $\mathrm{cm}^{3}$ Wasser umspült. Die Hinderung in die zusammengerollte Schreckstellung überzugehen sowie das feuchte Element waren Reiz genug, um die Tiere allmählich zur Entleerung ihrer Giftblasen (s. Abb. 3) zu veranlassen. Die erhaltenen gelben, wäßrigen Lösungen konnten nach Zusatz der dreifachen Menge Alkohol mit 2.4-DNP-Lösung gefällt oder zur Darstellung von Sekretlösungen mit Äther extrahiert werden.

\subsection{Identifizierung der Blaseninhaltsstoffe}

\subsubsection{Vorproben}

Der Geruch glich in allen Fällen dem des $p$-Benzochinons-1.4 und seiner niederen Homologen. Beim Verreiben mit etwas Hydrochinon nimmt das gelbe Sekret eine dunkle Farbe an, eine für Chinone charakteristische und wohlbekannte Reaktion.

Betüpfelt man einen Fleck des Sekrets auf einem Filterpapier mit etwas salzsaurer 2.4-DNP-Lösung, so kann man eine Verfärbung des ursprünglich gelben Flecks nach orangerot feststellen. In Ammoniakatmosphäre tritt die für Chinonmonohydrazone charakteristische Violettfärbung des orangeroten Flecks ein.

\subsubsection{UV-Absorption des Sekretes}

Der UV-Absorption kommt gerade bei den Diplopoden-Sekreten eine große Bedeutung zu. Die Aussagen, die sich im Wellenlängengebiet von 300 bis $400 \mathrm{~m} \mu$ machen lassen, können der Zuordnung

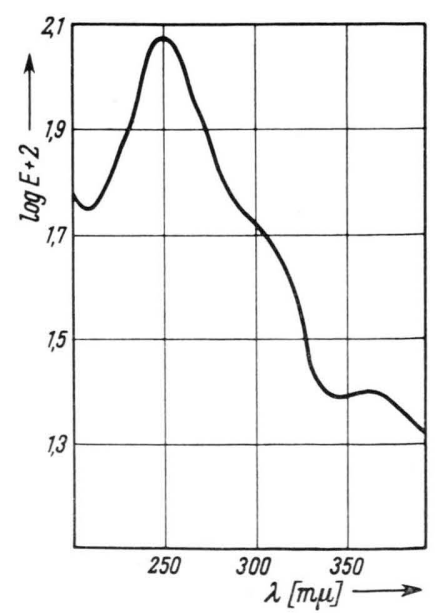

Abb. 4. UV-Absorptionskurve des Rohsekrets von Cylindroiulus teutonicus $\mathrm{P}$ o c o c k., in Äthanol. $\lambda_{\max }$ : 250 und $370 \mathrm{~m} \mu$.

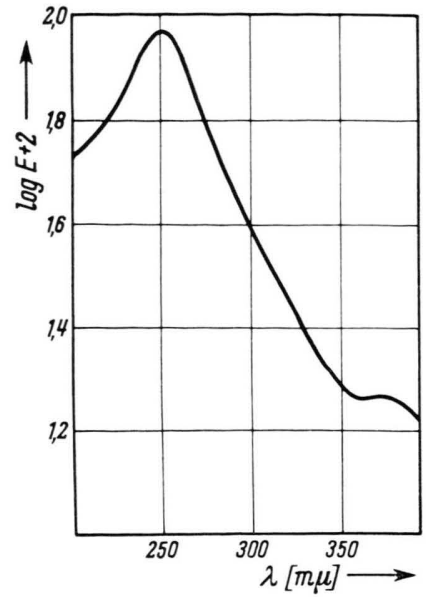

Abb. 5. UV-Absorptionskurve des Rohsekrets von Brachyiulus unilineatus Koch, in Äthanol. $\lambda_{\max }$ : 252 und $374 \mathrm{~m} \mu$.

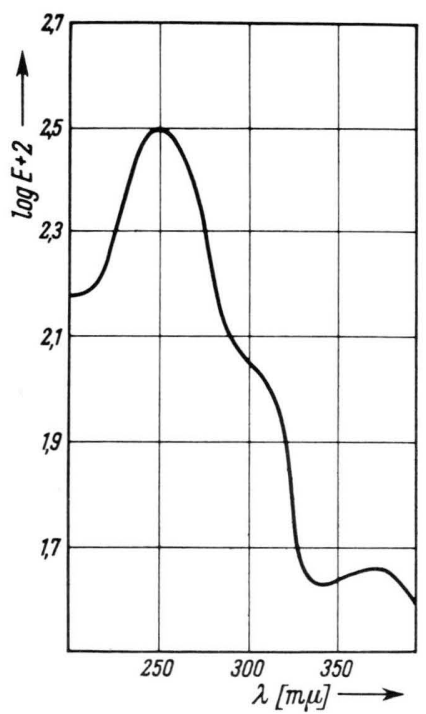

Abb. 6. UV-Absorptionskurve des Rohsekrets vom peruanischen Rhinocricus, in Äthanol. $\lambda_{\max }: 251$ und $371 \mathrm{~m} \mu$.

eines Chinons zu einer bestimmten Struktur dienen, denn nach Flaig und Mitarb. ${ }^{10}$ zeigen die UV-Spektren substituierter $p$-Benzochinone eine charakteristische Abhängigkeit der Lage der zweiten Maxima von der Art, Stellung und Anzahl der Substituenten.

Die in den Abb. 4 bis 9 wiedergegebenen UVSpektren der Diplopoden-Sekrete weisen ein Maximum im Bereich von $250-254 \mathrm{~m} \mu$ und ein zweites bei $370-375 \mathrm{~m} \mu$ auf.

Ein Vergleich mit den von Flaig und Mitarb. tabellierten Werten zeigt - wenn man einen gering-

10 W. Flaig u. J. Ch. Salfeld, Liebigs Ann. Chem. 618, 117 [1958]. 


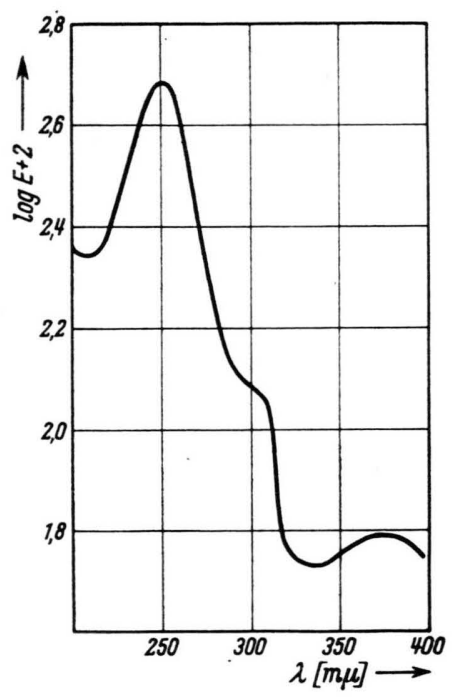

Abb. 7. UV-Absorptionskurve des Rohsekrets der unbekannten Art aus Madagaskar, in Äthanol. $\lambda_{\max }$ : 252 und $375 \mathrm{~m} \mu$.

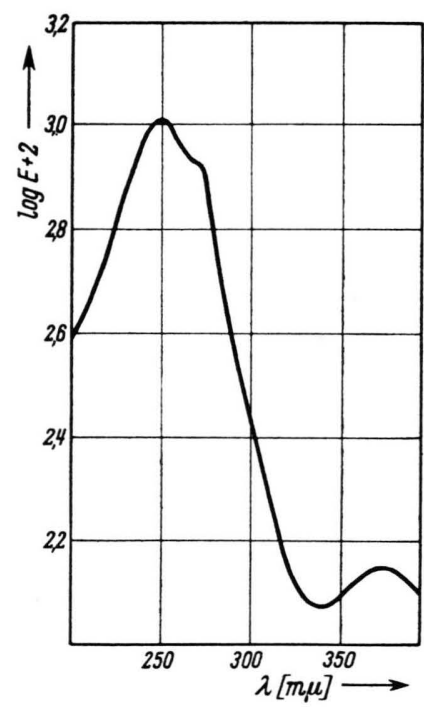

Abb. 8. UV-Absorptionskurve des Rohsekrets der unbekannten Art aus Belgisch Kongo, in $\ddot{\text { thanol. }} \lambda_{\max }: 250$ und $272 \mathrm{~m} \mu$.

fügigen Lösungsmitteleinfluß außer acht läßt eine Übereinstimmung dieser Meßwerte mit denen, die für das 2-Methyl-3-Methoxy-p-benzochinon-1.4 angegeben sind, nämlich $254 \mathrm{~m} \mu$ für das 1 . und $374 \mathrm{~m} \mu$ für das 2. Maximum. Damit gibt die UVSpektroskopie in den hier betrachteten Fällen neben der Bestätigung des Vorliegens von Chinon auch noch einen sehr deutlichen Hinweis auf die Art und Lage der möglichen Substituenten.

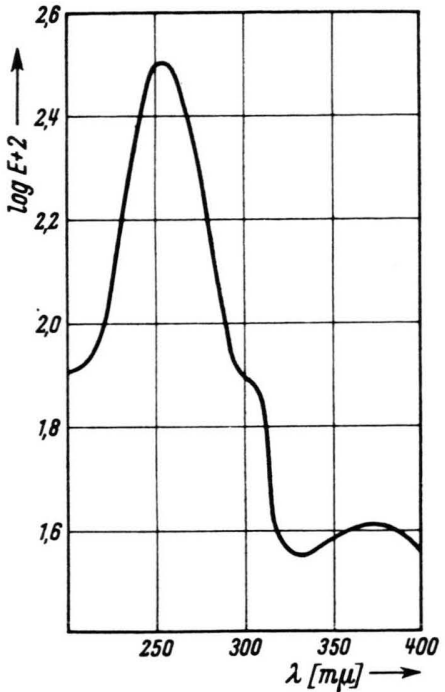

Abb. 9. UV-Absorptionskurve des Rohsekrets der unbekannten Art aus Neu-Guinea, in Äthanol. $\lambda_{\max }$ : 254 und $374 \mathrm{~m} \mu$.

\subsubsection{Aufarbeitung und Papierchromatographie der gefällten 2.4-Dinitrophenylhydrazone}

Die Rohprodukte der Fällung mit 2.4-DNP wurden abgenutscht und mit kaltem Wasser gewaschen. Aus den Mutterlaugen ließen sich die Reste gelöster Hydrazone durch Ausschütteln mit Chloroform gewinnen. Die Niederschläge und Extrakte zusammen ergaben schließlich die für die Vorreinigung an einer Kieselgelsäule benötigten Chloroformlösungen. In jedem einzelnen Fall bot sich das von den Tenebrioniden-Chinonen her gewohnte Bild ${ }^{11}$. Zuerst verließ das überschüssige Fällungsreagenz, als gelbe Zone wandernd, die Säule, gefolgt von unbedeutenden Mengen Bishydrazon, bis schließlich eine orangerote Zone aller Monohydrazone aufgefangen werden konnte. Diese Lösung diente direkt der papierchromatographischen Untersuchung. Ein Test ergab 3 von Chinon-Monohydrazon herrührende Flecken und brachte Aufschluß über das für die Entwicklung geeignetste Lösungsmittelsystem. Als solches erwies sich Chloroform/Benzol (1:1). Bei Verwendung von mit Formamid imprägniertem Papier wurde das Chromatogramm der Abb. 10 erhalten mit den in Tab. 1 aufgeführten $R_{f}$-Werten.

Es wurde aufgetragen:

Punkt 1 vorgereinigtes Hydrazongemisch der Diplopoden-Sekrete;

11 H. Schildknecht u. K. H. WeIs, Z. Naturforschg. 15 b, 757 [1960]. 


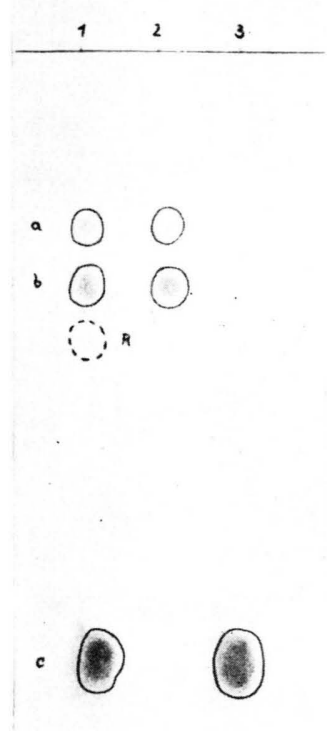

Abb. 10. Chromatogramme der aus den Diplopoden-Sekreten erhaltenen Monohydrazongemische. Papier: Schleicher \& Schüll 2043 b, Größe: 15 $40 \mathrm{~cm}$ (ab Startlinie). Art des Chromatogramms: absteigend. Imprägnierung: Formamid/Aceton (1:3). Entwicklung: Benzol/Chloroform ( $1: 1)$, in $\mathrm{NH}_{3}$. gesättigter Atmosphäre. Dauer der Entwicklung: 6,5 Stunden. $R=2.4$-DNP-Reagenz.

Punkt 2 Gemisch der isomeren Monohydrazone des 2-Methyl-p-benzochinon-1.4;

Punkt 3 Monohydrazon des 2-Methyl-3-methoxy-pbenzochinon-1.4.

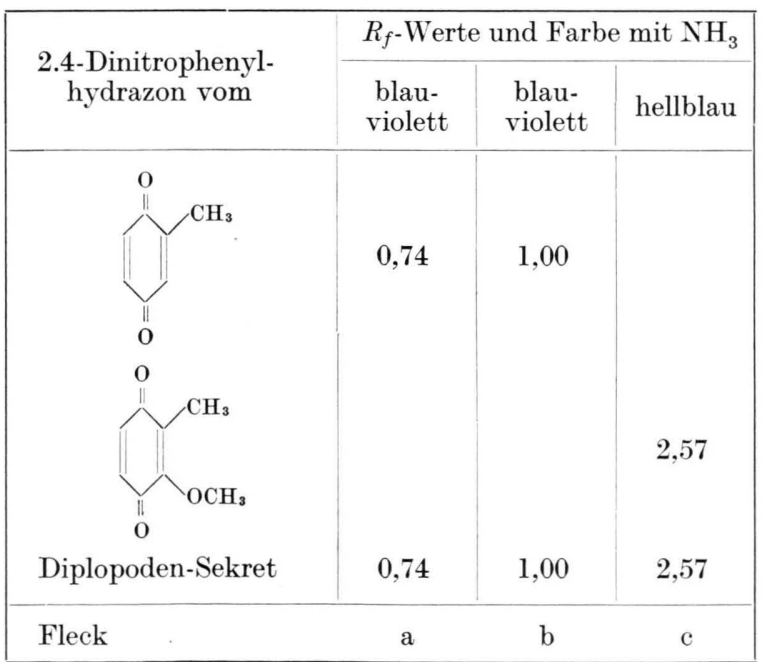

Tab. 1. $R_{f}$-Werte und Farbe der Hydrazone von Toluchinon, Methoxytoluchinon und des als Diplopodensekret erhaltenen Chinongemisches. Die $R_{f}$-Werte sind bezogen auf Toluchinonmonohydrazon (Fleck b).
Die Flecken a und b (Abb. 10) sind blauviolett gefärbt und schlagen nach gelb um, sobald das Chromatogramm aus der $\mathrm{NH}_{3}$-Atmosphäre entfernt wird. Es handelt sich um die folgenden isomeren Monohydrazone des 2-Methyl-p-benzochinon-1.4 ${ }^{12}$ :

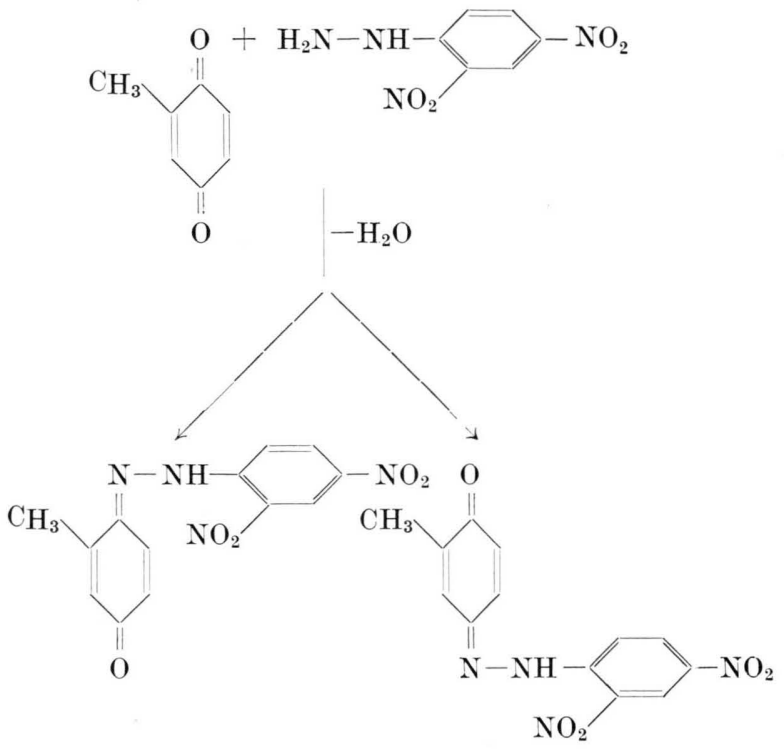

Fleck c weist eine bei hoher $\mathrm{NH}_{3}$-Konzentration hellblaue, sonst graue Färbung auf, die beim Fehlen von $\mathrm{NH}_{3}$ in gelb übergeht; er wird durch das Monohydrazon des 2-Methyl-3-methoxy-p-benzochinon-1.4 hervorgerufen.

Das Ergebnis der Papierchromatographie war, daß alle sechs der oben aufgeführten Diplopodenarten 2-Methyl-p-benzochinon-1.4 und 2-Methyl-3methoxy- $p$-benzochinon-1.4 in ihren Wehrsäften enthalten. Dagegen konnten an Hand der Chromatogramme der einzelnen Arten Unterschiede im Verhältnis der Mengen 2-Methyl-p-benzochinon-1.4 und 2-Methyl-3-methoxy- $p$-benzochinon-1.4 festgestellt werden, weshalb in jedem Falle dieses Mengenverhältnis mit Hilfe der UV-Spektroskopie ermittelt wurde.

Schneidet man aus dem erwähnten Papierchromatogramm gleiche Flächenanteile der Flecken b und c heraus, eluiert und spektroskopiert, so kann für jede untersuchte Diplopodenart das Verhältnis 2-Methyl-p-benzochinon-1.4 zu 2-Methyl-3-methoxy$p$-benzochinon-1.4 an Hand der Verhältnisse der maximalen Extinktion ermittelt werden. Der gering-

12 Über die Bildung der isomeren Hydrazone von substituierten Benzochinonen wird an anderer Stelle ausführlicher berichtet. 
fügige Fehler, der durch Verlust von wenig 2-Methylp-benzochinon-1.4 infolge Bildung des isomeren Monohydrazons (Fleck a) entsteht, fällt kaum ins Gewicht, weshalb auf ein Eluieren dieses Fleckes verzichtet wurde. Das Ergebnis ist in Tab. 2 aufgeführt.

\begin{tabular}{|c|c|c|}
\hline \multirow[b]{2}{*}{ Diplopodenart } & \multicolumn{2}{|c|}{$\begin{array}{c}\text { Mengenverhältnis } \\
\text { in } \%\end{array}$} \\
\hline & & \\
\hline Dinl a Belo Kono & 21 & 70 \\
\hline Dipl. a. Neu-Guinea & 17 & 83 \\
\hline Dipl. a. Madagaskar & 36 & 64 \\
\hline Rhinocricusart a. Peru & 48 & 52 \\
\hline Brachyiulus unilineatus & 49 & 51 \\
\hline Cylindroiulus teutonicus & 58 & 42 \\
\hline
\end{tabular}

Tab. 2. Quantitativer Vergleich des sezernierten Toluchinons und Methoxy-Toluchinons bei verschiedenen Diplopodenarten.

Zusätzlich zur papierchromatographischen Aussage über das Vorliegen von 2-Methyl-p-benzochinon-1.4- und 2-Methyl-3-methoxy-p-benzochinon-1.4Monohydrazonen in der 2.4-DNP-Fällung der Diplopodensekrete diente die UV-Spektroskopie und der Vergleich der Schmelzpunkte einer Sicherstellung dieses Ergebnisses. Die hierzu benötigten reinsten Hydrazone ließen sich durch Anwendung der präparativen Papierchromatographie (s. 3.1.) erhalten.

Die den Flecken b und $\mathrm{c}$ in Abb. 10 zugrunde liegenden Derivate der Diplopodensekrete zeigten sowohl hinsichtlich der Extinktion wie auch in der

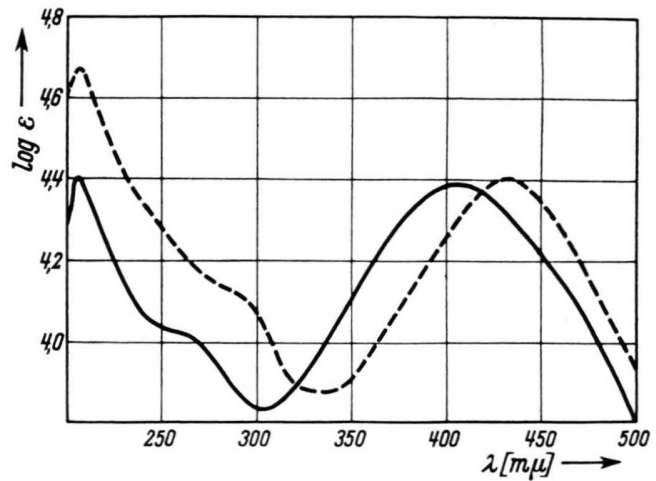

Abb. 11. UV-Absorptionsspektren der 2.4-DNP von Toluchinon $\left(\lambda_{\max }: 204\right.$ und $408 \mathrm{~m} \mu ; \log \varepsilon 4,40$ und 4,37) und 2-Methyl-3-Methoxy-p-benzochinon $--\left(\lambda_{\max }: 205\right.$ und $428 \mathrm{~m} \mu ; \log \varepsilon 4,69$ und 4,39), gemessen in Athanol.
Lage der Maxima volle Übereinstimmung mit den Monohydrazonen des 2-Methyl-p-benzochinon-1.4 bzw. des 2-Methyl-3-methoxy-p-benzochinon-1.4. Bemerkenswert ist die Verschiebung des 2. Maximums infolge Substitution durch die Methoxylgruppe (s. Abb. 11).

Auch die Schmelzpunkte der Sekretderivate stimmten mit den korrespondierenden Chinonmonohydrazonen vollkommen überein.

\subsubsection{Die je Individuum in den Blasen gespeicherte Chinonmenge}

Der bei der Reinigung der rohen 2.4-Dinitrophenylhydrazone an einer Kieselgelsäule nach Verdampfen des Lösungsmittels hinterbleibende HydrazonAnteil gibt Aufschluß über die von den Tieren sezernierte Chinonmenge. Für diese Näherungsbetrachtung wurde ein "mittleres Mol.-Gew.“ von 317 für das Hydrazongemisch und ein ebensolches von 137 für das zugrunde liegende Chinongemisch der Sekrete in Rechnung gesetzt. Durchschnittswerte konnten jedoch lediglich bei den einheimischen Diplopoden ermittelt werden:

10 Exemplare von Cylindroiulus teutonicus gaben $28 \mathrm{mg}$ Hydrazongemisch, das entspricht einer Chinonmenge von 1,2 mg je Individuum.

5 Exemplare von Brachyiulus unilineatus gaben $6,5 \mathrm{mg}$ Hydrazongemisch, was einer Chinonmenge von $0,56 \mathrm{mg}$ je Individuum entspricht.

Bei den großen, tropischen Arten konnten exakte Resultate deshalb nicht erwartet werden, weil die Tiere stets nur einzeln und nur für ganz kurze Zeit zur Verfügung standen. Über den Füllungsgrad der Blasen ließen sich daher nur Vermutungen anstellen. Immerhin sei erwähnt, daß die von einem dieser großen Tausendfüßler erhaltene Hydrazonmenge $500-800 \mathrm{mg}$ betrug, was Chinonmengen von etwa $200-350 \mathrm{mg}$ entsprechen würde.

\section{Experimenteller Teil}

\subsection{Feinreinigung von Chinon-Monohydrazonen durch präparative Papierchromatographie}

Die an der Kieselgelsäure weitgehend vorgereinigten Hydrazongemische der Diplopoden-Sekrete wurden zur Feinreinigung und Auftrennung in die einzelnen Komponenten in Chloroform bis zur Sättigung gelöst und am Start eines $20 \mathrm{~cm}$ breiten und $40 \mathrm{~cm}$ langen, formamidimprägnierten Papierstreifens aufgetragen, und zwar in Form eines 3 bis $5 \mathrm{~cm}$ breiten Bandes. Im System Benzol/Chloroform (1:1), 6 Stdn. lang entwickelt, zeigten sich 5 quer zur Laufrichtung liegende Streifen, von denen 3 (in $\mathrm{NH}_{3}$-Atmosphäre) violett waren. Mit 
der Front lief das gelbe Fällungsreagenz, gefolgt vom grauvioletten 2-Methyl-3-methoxy-p-benzochinon-1.4Monohydrazon. Daran anschließend erschien ein wiederum gelbes Derivat des Fällungsreagenz gefolgt von den beiden hellblauen Streifen des 2-Methyl-p-benzochinon-1.4-Monohydrazones, von denen das Monohydrazon II die Hauptmenge ausmachte. Das lufttrockene Chromatogramm wurde in die entsprechenden Streifen zerschnitten, diese mit Methylenchlorid eluiert und die Eluate zur Befreiung von Papierfasern und gelöstem Formamid auf eine kleine Kieselgelsäule aufgebracht. Das Formamid blieb im Säulenkopf hängen und die aufgefangenen, orangefarbenen Zonen ergaben im
Falle der Chinon-Monohydrazone nach langsamem Eindunsten des Lösungsmittels hellrote, nadelige Kristalldrusen. Die Ausbeute an kristallisierten Monohydrazonen betrug je Chromatogramm und Streifen etwa 1 bis $3 \mathrm{mg}$ der reinen Verbindungen.

Der Deutschen Forschungsgemeinschaft danken wir für die Unterstützung dieser Arbeit, Herrn Prof. Dr. H.-J. Stammer, Herrn Dr. G. Osche (Institut für Zoologie der Universität Erlangen) und Herrn Dr. O. Kraus (Senckenbergis che N a t urforschende Gesellschaft) für die Bestimmung und Überlassung von Diplopodenarten und für wertvolle Diskussion.

\title{
Der Sauerstoffverbrauch und der Gehalt an Ca, Cu und einigen anderen Spurenelementen bei terrestrischen Asseln
}

\author{
Von Wolfgang Wieser \\ Aus dem 2. Zoologischen Institut der Universität Wien \\ und Hans Makart, \\ Mineralogisches Institut der Universität Wien* \\ (Z. Naturforschg. 16 b, 816-819 [1961] ; eingegangen am 25. Mai 1961)
}

\begin{abstract}
The oxygen consumption and the content of $\mathrm{Ca}, \mathrm{Cu}, \mathrm{K}, \mathrm{Sr}, \mathrm{Zn}, \mathrm{Fe}, \mathrm{P}$ and $\mathrm{Mg}$ was determined in several terrestric isopods. Porcellio scaber, Syspastus brevicornis and the three species of Armadilli. dium investigated display significant differences in their calcium contents and there is an inverse relationship between the latter and the oxygen consumption of the animals at $20^{\circ} \mathrm{C}$. The $Q_{\mathrm{O}_{2}}$ of $S$. brevicornis is $13 \pm 3.5 \mathrm{~mm}^{3} / \mathrm{g} / \mathrm{hr}$, the lowest ever recorded for a crustacean at $20^{\circ} \mathrm{C}$. The copper content of the isopods is high and suggests the presence of hemocyanin. It is highest in a population of P. scaber from Cornwall, England, where it amounts to approximately $0,1 \%$ of the dry weight of the animals. Most of the copper is present in the four hepatic tubules (hepatopancreas) which, in one sample from the population mentioned above, contained $1,4 \%$ copper, the highest value ever found in an invertebrate. The $Q_{\mathrm{O}_{2}}$ of animals with more or less filled gut seems to be higher in the population with the high copper content, whereas in animals with half empty or empty gut the oxygen consumption is the same in the three populations of $P$. scaber studied.
\end{abstract}

Die elementare Zusammensetzung terrestrischer Crustaceen scheint bisher noch nicht quantitativ und systematisch untersucht worden zu sein. Dies steht im Gegensatz zu den zahlreichen Untersuchungen an marinen Crustaceen (Zusammenfassung ${ }^{1,2}$ ). Analysen dieser Art können zu weitreichenden Aufschlüssen über die Leistungen und die Struktur der Tiere sowie über ihre Umwelt führen.

Mittels der röntgenfluoreszenz-analytischen Methode wurde bei einigen terrestrischen Isopoden (Porcellio scaber Latr., Armadillidium vulgare (Latr.), A. granulatum Brandt, A. klugii Brandt, Syspastus brevicornis Ebner und Cylisticus con-

* Jetzige Adresse: Metallwerke Dornach AG., Dornach, Schweiz.

1 A. P. Vinogradov, The elementary chemical composition of marine organisms. Sears Found. Mar. Res. Mem. 2 [1953]. vexus $\mathrm{B}$. L.) der Gehalt an $\mathrm{Ca}, \mathrm{K}, \mathrm{Sr}, \mathrm{Zn}, \mathrm{Cu}$ und $\mathrm{Fe}$ bestimmt. Außerdem wurden in einigen Fällen $\mathrm{P}$ auf spektralphotometrische und $\mathrm{Mg}$ auf naßchemische Weise bestimmt.

Besonderes Augenmerk wurde auf den Ca- und den $\mathrm{Cu}-\mathrm{Gehalt}$ gelegt. Was das Ca betrifft, so war nachzuprüfen, ob eine Beziehung zwischen dem Kalkgehalt der Kutikula der einzelnen Arten und ihrer systematischen Stellung, bzw. ihrer Lebensweise besteht. Der Cu-Gehalt hinwiederum erschien deshalb interessant, weil bei Isopoden noch niemals Hämocyanin nachgewiesen worden ist $^{3}$, obwohl es

2 G. D. Nicholls, H. Curl u. V. T. Bowen, Limnol. Oceanogr. 4, 472 [1959].

3 T. W. Goodwin, Biochemistry of Pigments, in: T. H. WAterman (Ed.), The Physiology of Crustacea, Bd. 1, S. 101. Academic Press, Inc., New York und London 1960. 